La revue La revue pour l'histoire du CNRS

POUR L'HISTOIRE DU CNRS $\quad 15 \mid 2006$

CNRS et Université

\title{
La mobilité en sciences humaines et sociales
}

L'Institut d'histoire moderne et contemporaine

\section{Christophe Charle}

\section{(2) OpenEdition}

Journals

Édition électronique

URL : https://journals.openedition.org/histoire-cnrs/515

DOI : 10.4000/histoire-cnrs.515

ISSN : 1955-2408

Éditeur

CNRS Éditions

Édition imprimée

Date de publication : 3 novembre 2006

ISBN : 978-2-271-06452-3

ISSN : $1298-9800$

Référence électronique

Christophe Charle, "La mobilité en sciences humaines et sociales ", La revue pour l'histoire du CNRS

[En ligne], 15 | 2006, mis en ligne le 23 novembre 2008, consulté le 20 mai 2021. URL : http://

journals.openedition.org/histoire-cnrs/515; DOI : https://doi.org/10.4000/histoire-cnrs.515

Ce document a été généré automatiquement le 20 mai 2021

Comité pour l'histoire du CNRS 


\title{
La mobilité en sciences humaines et sociales
}

\author{
L'Institut d'histoire moderne et contemporaine
}

Christophe Charle

1 En 1999, le laboratoire est devenu une unité mixte CNRS/École normale supérieure (UMR 8066). L'équipe s'est notoirement étoffée (plus de trente personnes en comptant les associés et les post-docs) et diversifiée en termes de catégories (chercheurs, enseignants chercheurs, enseignants chercheurs associés, chercheurs associés, doctorants et post doctorants) et de types de travaux. Surtout, le personnel s'est presque entièrement renouvelé au fil du temps si bien qu'aucun des chercheurs «fondateurs» ne se retrouve dans les listes actuelles. L'auteur de ces lignes est le dernier survivant de la première époque : même s'il n'a pas été rattaché dès 1979, première année d'activité effective, il est arrivé volontairement en 1980 et a donc connu les générations successives ce qui justifie l'entreprise de cette histoire du temps présent qui n'est pas sa spécialité officielle ${ }^{1}$. Avec cet exemple, nous voudrions aussi réagir contre une certaine sinistrose qui caractérise les tableaux récents de la recherche française. La crise de l'année 2003-2004 a attiré l'attention sur un certain nombre de dysfonctionnements de la recherche publique, mais a finalement abouti à négliger certaines des évolutions positives qui sont intervenues malgré ces problèmes.

La situation initiale

2 Les modes scientifiques contemporaines sont plutôt hostiles à la notion de laboratoire propre sauf lorsqu'il existe de grands projets pluriannuels et de gros équipements à faire tourner indépendamment des contraintes de l'enseignement supérieur. On peut donc s'étonner que l'idée de laboratoire propre ait pu s'appliquer en histoire où le mode de travail individualiste et la légèreté de l'appareillage technique ont très longtemps caractérisé le "métier» d'historien. Les projets initiaux de l'IHMC ont reposé sur des enquêtes de longue durée et la constitution de banques de données informatisées impliquant donc des personnels à temps plein. Tous les chercheurs présents dans cette phase inséraient leur projet individuel dans une enquête collective du laboratoire: la prosopographie des élites avait été sélectionnée comme l'un des 
thèmes fédérateurs de l'unité. En ont résulté l'implication de Denis Woronoff, plus tard de Dominique Barjot, dans l'enquête sur le patronat du Second Empire, en parallèle de leurs propres travaux sur l'histoire de deux branches industrielles, respectivement la sidérurgie à l'époque de la Révolution et de l'Empire, les entreprises de travaux publics à l'époque contemporaine. De même, un autre chercheur de la première génération, Michel-Edmond Richard, travaillait à la fois à sa thèse sur les notables protestants du premier XIX ${ }^{e}$ siècle sous la direction de Jean-Marie Mayeur et à l'enquête sur le personnel parlementaire menée en coopération avec le Centre de recherches en histoire du XIX ${ }^{e}$ siècle des universités Paris I et Paris IV ${ }^{2}$. L'auteur de ces lignes préparait conjointement sa thèse d'État sur les Intellectuels et élites de 1880 à 1900 et produisait, seul, puis en collaboration avec un ingénieur du laboratoire, Eva Telkes, sa prosopographie des universitaires français donnant lieu à quatre dictionnaires et à deux colloques. Ces prosopographies n'étaient pas conçues comme des enquêtes isolées mais comme les têtes de réseau à maillage national voire international : les travaux sur le patronat faisaient coopérer des chercheurs du laboratoire et des équipes universitaires en province (Caen, Marseille, Dijon, Lille, etc.) pour confectionner un certain nombre de volumes régionaux et des synthèses fondées sur des colloques rassemblant les diverses équipes. Il en allait de même pour le projet sur le Refuge huguenot animé par Michèle Magdelaine, recrutée au laboratoire en 1985 et dont la banque de données était enrichie par des correspondants en Angleterre, en Allemagne, en Hollande et en Suisse.

Partir, rester?

3 Cet engagement lourd, individuel et collectif, contraste avec les obligations assez légères des attachés ou chargés de recherche dans la période précédente. Recrutés trois ou quatre ans pour avancer leur thèse d'État, ils étaient censés repartir assez vite à l'Université comme assistant, maître assistant ou chargé de cours. L'autre originalité de cette première génération est d'avoir effectué un parcours relativement long au sein du laboratoire comme le permettait dorénavant le vote du nouveau statut des chercheurs introduisant la titularisation comme fonctionnaires ${ }^{3}$. L'on ne compte plus les textes, officiels ou non, déplorant le «manque de mobilité »des chercheurs du CNRS pendant cette période. Effectivement, l'incitation à déposer des candidatures auprès des universités a beaucoup baissé dans les années 1980 dans la mesure où, dans le même temps, les conditions de travail s'y dégradaient du fait de la seconde vague de massification de la fin des années 1980.

Pourtant près de la moitié des chercheurs de l'IHMC restent mobiles au long de la période d'observation (1978-1999) ${ }^{4}$. Cette mobilité est indépendante du grade atteint: les directeurs de recherche et les chargés de recherche de première classe y figurent. Le même mouvement touche les directeurs de l'IHMC : un seul (Daniel Roche) reste plus de cinq ans en fonction.

5 Conformément là aussi à un discours qu'on entend beaucoup au CNRS, une première hypothèse simple d'explication pourrait être la lenteur des promotions en sciences humaines et sociales comparée aux carrières universitaires. Ce blocage inciterait certains chercheurs docteurs ès lettres ou habilités à sauter le pas plus facilement. Or, parmi les sortants, tous docteurs d'État il est vrai, on trouve aussi bien des chargés de recherche de $1^{\text {re }}$ classe, qui conquièrent ainsi une promotion en devenant professeur, que des directeurs de recherche de deuxième classe qui, dans un premier temps, n'en tirent pas d'avantage financier particulier: face à quatre chargés de recherche ${ }^{5}$, on 
trouve trois directeurs de recherche ${ }^{6}$. On peut ajouter à cette seconde cohorte Frédéric Barbier qui a effectué, en tant que directeur de recherche, deux mobilités hors laboratoire: l'une à Goettingen (deux ans) à la Mission historique française en Allemagne, l'autre (quatre ans) comme professeur à l'ENSSIB. En outre, ces partants vers l'Université se sont fait élire au moment précis où la surcharge des effectifs et l'obligation pour la plupart de quitter Paris (pour Caen, Bordeaux, Lille, Lyon) ajoutaient des charges de travail et financières (pour ceux qui continuaient d'habiter la capitale) non négligeables. L'ambition (légitime) ne rend donc compte que partiellement de l'appel vers l'extérieur. Il serait tentant alors de recourir à une explication de type caractérologique opposant chercheurs devenus universitaires et chercheurs restés chercheurs: les uns, plus généralistes, auraient le goût du monde extérieur, les autres, plus spécialistes, se replieraient dans leur tour d'ivoire. En fait, modernistes et contemporanéistes sont également présents chez les mobiles (quatre contemporanéistes pour trois modernistes). En revanche l'une des caractéristiques communes aux chercheurs fidèles à l'IHMC est leur entrée relativement tardive dans les cadres de la recherche (au-delà de 40 ans), après parfois de longues carrières dans l'enseignement secondaire, l'administration ou à l'étranger. Pour eux, l'accès au CNRS prend de ce point de vue une autre signification : ce n'est pas un stage dans un parcours mais le couronnement d'une trajectoire attirée par la recherche mais longtemps frustrée de ne pas s'y adonner du fait de charges familiales ou professionnelles. On compte en particulier dans ce groupe, ce n'est pas un hasard, deux femmes mariées et mères de famille élues au CNRS nettement plus tard que leurs homologues masculins, à 48 et 42 ans.

6 À cette explication plutôt sociale, s'ajoutent aussi des considérations scientifiques. L'un des handicaps de la position de directeur de recherche, en particulier en sciences humaines et sociales, est la difficulté pour encadrer véritablement des travaux d'étudiant. La plupart des étudiants de thèse ou de DEA s'adressent en général à des enseignants- chercheurs pour les guider. Cette coupure par rapport aux jeunes générations était particulièrement ressentie dans un laboratoire propre, par définition indépendant de l'environnement universitaire (à la différence des anciennes URA ou actuelles UMR) et était accentuée avec son implantation à l'École normale supérieure, établissement dont les élèves sont doublement encadrés par un corps enseignant interne et les professeurs des universités (parisiennes) où ils sont inscrits. L'espace ouvert aux chercheurs pour former les jeunes apprentis chercheurs se retrouvait ainsi des plus réduits.

7 Le dernier élément d'explication renvoie aussi à la logique des enquêtes collectives dans lesquelles étaient inscrits ces chercheurs partants. Après la vague des travaux prosopographiques et la confection des instruments de travail qui caractérisent la première décennie de l'IHMC et auxquels les chercheurs mobiles ont participé activement, le changement de conjoncture historiographique des années 1990 se manifeste là aussi : la crise de l'édition scientifique rend problématique ce type de publication, avant que la mise en ligne, dix ans plus tard, n'offre une alternative plus viable économiquement. La montée en puissance de l'histoire des représentations qui redonne vie aux travaux individuels réduit les prestiges de l'histoire économique et sociale même affinée par la biographie collective qui dominait dans les premières enquêtes. L'atomisation grandissante de la communauté des historiens et le remplacement de la grosse informatique par la micro-informatique sapent les bases $\mathrm{du}$ consensus intellectuel et technique sur ce que peut être un instrument de travail 
collectif et la dépendance par rapport aux centres de calcul de la première époque. Tous ces facteurs internes et externes rendent compte des modalités de la mobilité du CNRS vers l'Université propre à l'IHMC.

8 Cet exemple limité autorise aussi à avancer une conclusion plus générale : la mobilité ne se décrète pas, mais des conditions historiques et conjoncturelles pèsent plus qu'ailleurs peut-être en sciences humaines et sociales. Plus on recrute les chercheurs tard, moins ils risquent de changer de métier, plus ils bloquent le rajeunissement nécessaire de la pyramide par la suite. En second lieu, l'enseignement supérieur peut attirer, s'il n'est pas identifié uniquement aux aspects les plus ingrats de la formation mais perçu comme le lieu de contact privilégié avec la relève potentielle de la recherche. C'était le cas il y a une dizaine d'années. Ce n'est peut-être plus aussi vrai aujourd'hui où le système LMD incite les étudiants à privilégier de plus en plus les formations master professionnelles et où la restriction des bourses de thèse et des débouchés prévisibles pour les docteurs n'incitent pas les apprentis chercheurs à l'optimisme, comme le montre la multiplication des associations de doctorants et des mouvements de lutte contre la précarité. Peu d'universités disposent également de structures de recherche en sciences humaines et sociales dotées des moyens, de la reconnaissance scientifique et de stratégies thématiques pour rivaliser vraiment avec les unités originaires du CNRS. Les chercheurs qui ont besoin de moyens substantiels parce qu'ils travaillent, par exemple, sur des aires lointaines ou veulent monter des réseaux internationaux y regarderont sans doute à deux fois avant de lâcher la proie d'un budget de mission pour l'ombre de disciples potentiels mais forcément rares. Mais le plus grave est sans doute ailleurs : la rétraction simultanée des postes à l'entrée au CNRS et dans les universités fait monter les réactions corporatives des deux côtés. Chaque corps tend à tenir un discours de la jalousie et du ressentiment sur l'autre, au détriment des échanges et des coopérations, donc de la mobilité. C'était moins vrai à l'époque considérée, même si des signes avant-coureurs étaient déjà présents. Il convient que les nouvelles structures qui se mettent en place depuis le vote de la loi sur la recherche prennent absolument en compte ces questions sous peine d'une ossification supplémentaire des personnels de l'enseignement supérieur et de la recherche, préjudiciable à tous et surtout à la productivité scientifique. À travers cette monographie, c'est donc aussi un message d'espoir que l'on voudrait proposer: pour peu que les individus se mobilisent et saisissent les occasions les institutions de recherche ne sont pas toujours vouées à l'obsolescence ou au vieillissement. 


\begin{tabular}{|c|c|}
\hline \multicolumn{2}{|c|}{$\begin{array}{l}\text { PROSOPOGRAPHIE COMPAREE DES CHERCHEURS DE L'IHMC (1979-99) } \\
\text { (classement par date d'arrivée dans l'unité) }\end{array}$} \\
\hline CHERCHEURS PASSÉS A L'UNIVERSITÉ & CHERCHEURS * STABLES * \\
\hline \multirow{2}{*}{$\begin{array}{r}\text { Denis Woronoff (1939) } \\
\text { - IHMC : 1979-87 - Docteur es lettres (1981) Maítre de recherche: } 1982 \\
\text { Date de départ : } 1987 \text { (CRH) } \\
\text { - Professeur des universités Paris I (1992) }\end{array}$} & $\begin{array}{l}\text { Michel-Edmond Richard (1926) } \\
\text { • IHMC: 1979-91 • Retraité: 1991, chargé de recherches }\end{array}$ \\
\hline & $\begin{array}{l}\text { Jean Nagle (1930) } \\
\text { IHMC: } 1979-95 \bullet \text { Retraité: } 1995 \text {, chargé de recherches } \\
\end{array}$ \\
\hline $\begin{array}{r}\text { André Zysberg (1947) } \\
\text { IHMC : } 1980-89 \text { • Docteur és lettres, 1986 } \\
\text { - Professeur des universités: } 1989 \text { (Caen) }\end{array}$ & $\begin{array}{l}\text { Gerhard Fries (1937) } \\
\text {-IHMC: } 1979-2002 \bullet \text { Retraité: } 2002 \text {, chargé de recherches }\end{array}$ \\
\hline $\begin{array}{r}\text { Christophe Charle (1951) } \\
\text { - IHMC : 1980-1991 • Docteur es lettres (1986) } \\
\text { • Directeur de recherche:1986 } \\
\text { - Professeur des universités: } 1991 \text { (Lyon III, puis Paris-I: } 1993 \text { ) } \\
\end{array}$ & $\begin{array}{l}\text { Frédéric Barbier (1952) } \\
\text { - IHMC: } 1982 \text { • Docteur ès lettres (1987) • Directeur de recherche } \\
\text { - Directeur d'études a l'EPHE } \\
\text { - Professeur à l'ENSSIB (2000-2004) }\end{array}$ \\
\hline \multirow{2}{*}{$\begin{array}{r}\text { Emmanuel Chadeau (1956) } \\
\bullet \text { IHTP puis IHMC (1985-88) • Docteur és lettres (1986) } \\
\text { • Professeur des universités: } 1988 \text { (Lille III) } \\
\end{array}$} & $\begin{array}{l}\text { Michelle Magdelaine (1933) } \\
\text { - IHMC: 1985-99 • Retraitée: 1999, chargée de recherches }\end{array}$ \\
\hline & $\begin{array}{l}\text { Jacques Bottin (1946) } \\
\text {-IHMC: } 1986 \bullet \text { HDR: 1998 • Directeur de recherche }\end{array}$ \\
\hline $\begin{array}{r}\text { Alain Cabantous (1946) } \\
\text { - Docteur ès lettres (1987) - IHMC: 1987-95 • Directeur de recherche: } 1993 \\
\text { • Professeur des universités (Paris X): 1995, Paris-1: } 1998 \\
\end{array}$ & $\begin{array}{l}\text { Nicole Pellegrin (1944) } \\
\text {-IHMC: } 1986 \text { • Chargee de recherches } \\
\end{array}$ \\
\hline \multirow{2}{*}{$\begin{array}{r}\text { Dominique Barjot (1950) } \\
\text { •IHMC: } 1988-90 \bullet \text { Docteur es lettres (1989) } \\
\text { • Professeur des universités: } 1990 \text { (Caen), Paris-IV } \\
\end{array}$} & $\begin{array}{l}\text { Andrée Corvol (1947) } \\
\text { Docteur ès lettres (1983) •IHMC: } 1989 \bullet \text { Directeur de recherche }\end{array}$ \\
\hline & $\begin{array}{l}\text { Muriel Le Roux (1961) } \\
\bullet \text { IHMC: } 1996 \bullet \text { Docteur en histoire • Chargée de recherches }\end{array}$ \\
\hline $\begin{array}{l}\text { Pascal Griset (1957) } \\
\text { - IHMC: } 1988-96 \text { • Docteur en histoire: } 1993 \cdot \text { HDR: 1994 } \\
\text { - Professeur des universités: } 1996 \text { (Bordeaux III) Paris-IV }\end{array}$ & 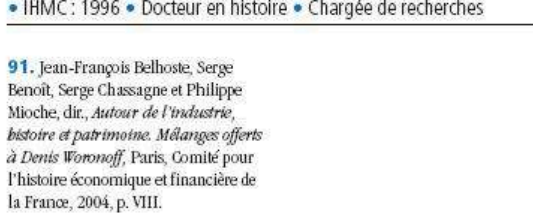 \\
\hline
\end{tabular}

\section{NOTES}

1. Les données citées s'appuient sur les dossiers personnels conservés dans les archives du laboratoire, sur les divers rapports d'activité et les annuaires d'historiens disponibles.

2. Michel-Edmond Richard, Notables protestants en France dans la première moitié du XIX ${ }^{e}$ siècle, Caen, Éd. du Lys, 1996 ; Les Immortels du Sénat, 1875-1918 : les cent seize inamovibles de la Troisième République, sous la direction de Jean- Marie Mayeur et Alain Corbin ; avec le concours d'Arlette Schweitz, Paris, Publications de la Sorbonne, 1995.

3. Cf. annexe prosopographique : les chercheurs mobiles restent entre trois et onze ans dans l'unité.

4. Ce terminus ad quem correspond à la fin de la direction de Daniel Roche que l'auteur de ces lignes a remplacé et au passage au statut d'UMR qui modifie notablement les équilibres entre les diverses catégories de personnel par l'intégration de trois enseignants-chercheurs de l'École normale supérieure. Il paraît aussi de bonne déontologie et de bonne méthode de ne pas inclure les années où l'auteur est juge et partie et surtout où la logique du laboratoire propre s'efface progressivement.

5. D. Barjot, E. Chadeau, P. Griset, A. Zysberg.

6. A. Cabantous, C. Charle, D. Woronoff (ce dernier a d'abord changé de laboratoire avant d'être élu à l'université de Paris I en 1992). 


\section{RÉSUMÉS}

Retracer l'évolution des caractéristiques des chercheurs d'un laboratoire de sciences humaines et sociales, comme l'Institut d'histoire moderne et contemporaine (IHMC), peut sembler une entreprise limitée compte tenu de la taille modeste de ce laboratoire fondé en 1978. Le projet original s'inscrivait dans la politique volontariste du CNRS destinée à structurer l'histoire postmédiévale, comme l'avaient été la sociologie et l'économie, autour de laboratoires généralistes aux fonctions distinctes des centres de recherche universitaires voués à une spécialisation grandissante et qui souffraient du manque de moyens. Près de trente ans plus tard, le paysage scientifique a radicalement changé, tout comme la physionomie et la fonction de l'IHMC.

It is difficult to summarize the evolutions of the characteristics of researchers in human and social sciences belonging to such a small lab like the Modern and contemporary history institute (IHMC), founded in 1978. This original project was born in a context of a voluntarist policy from the CNRS to reorganize post-medieval history, as it had been done with sociology and economy, with new labs, distinct from university research centers, which were appointed to become more specialised but with less financial means. Almost thirty years later, the scientific landscape totally changed, so as the function of the IHMC.

\section{AUTEUR}

\section{CHRISTOPHE CHARLE}

Christophe Charle, professeur d'histoire contemporaine à l'université de Paris IPanthéon- Sorbonne, est directeur de l'Institut d'histoire moderne et contemporaine (CNRS/École normale supérieure) et membre de l'Institut universitaire de France. 